Supporting experimental evidence for

\title{
Borane-catalyzed Hydrosilylation of Thiobenzophenone: A New Route to Silicon- Sulfur Bond Formation
}

Daniel J. Harrison ${ }^{a}$, Robert McDonald ${ }^{b}$ and Lisa Rosenberg ${ }^{a}$

${ }^{a}$ Department of Chemistry, University of Victoria, P.O. Box 3065, Victoria, British Columbia, CANADA V8W $3 V 6$

${ }^{\mathrm{b}} X$-Ray Crystallography Laboratory, Department of Chemistry, University of Alberta, Edmonton, AB, Canada T6G 2G2. Fax: 017804928231 Tel: 01780492 2485; E-mail: Bob.McDonald@ualberta.ca

General. Experiments were conducted in an inert $\left(\mathrm{N}_{2}\right)$ atmosphere using standard glovebox and Schlenk techniques. Hexanes, benzene and benzene- $\mathrm{d}_{6}$ were dried over $\mathrm{Na} /$ benzophenone and distilled prior to use. Triphenylsilane, methyldiphenylsilane, dimethylphenylsilane, triethylsilane, 1,1,2,2tetramethyldisilane, 1,1,2,2-tetraisopropyldisilane and benzophenone were purchased from Aldrich and used without further purification. 1,1,2,2-Tetraphenyldisilane ${ }^{1}$, thiobenzophenone ${ }^{2}$, and $\mathrm{B}\left(\mathrm{C}_{6} \mathrm{~F}_{5}\right)_{3}{ }^{3}$ were synthesized according to literature procedures. ${ }^{1} \mathrm{H}$ and ${ }^{13} \mathrm{C}\left\{{ }^{1} \mathrm{H}\right\}$ NMR spectra were obtained on Bruker AMX 250 or AMX 300 spectrometers. Residual proton peaks were used as reference: ${ }^{1} \mathrm{H}(\delta$, ppm, benzene- $\left.\mathrm{d}_{6}, 7.16\right)$; and ${ }^{13} \mathrm{C}\left(\delta\right.$, ppm, benzene- $\left.\mathrm{d}_{6}, 128.39\right) .{ }^{29} \mathrm{Si}\left\{{ }^{1} \mathrm{H}\right\}$ NMR spectra were recorded on a Bruker AV $500 \mathrm{MHz}$ instrument using an INEPT pulse sequence $\left({ }^{1} \mathrm{~J}_{\mathrm{SiH}}=185 \mathrm{~Hz},{ }^{2} \mathrm{~J}_{\mathrm{SiH}}=7 \mathrm{~Hz},{ }^{3} \mathrm{~J}_{\mathrm{SiH}}=3.5\right.$ $\mathrm{Hz}$ ), and referenced to external TMS at $0 \mathrm{ppm}$. IR spectra were recorded on a Perkin Elmer Spectrum 1000 FT-IR spectrometer using $\mathrm{NaCl}$ solution cells. UV-vis data was obtained using a Varian Cary 50 UV-vis spectrophotometer. Low resolution mass spectrometry data were obtained using a Kratos Concept $\mathrm{H}$ spectrometer. Microanalyses were performed by Canadian Microanalytical Service, Delta, BC, Canada.

\section{Synthesis of samples of silylthioethers 1-6 for purposes of characterization:}

1: Triphenylsilane (261 mg, $1.00 \mathrm{mmol})$ and thiobenzophenone (199 $\mathrm{mg}, 1.00 \mathrm{mmol})$ were combined with toluene $(2 \mathrm{~mL})$. To the resulting strongly blue-colored solution, $\mathrm{B}\left(\mathrm{C}_{6} \mathrm{~F}_{5}\right)_{3}(20 \mathrm{mg}, 0.040 \mathrm{mmol}, 4$ mol \%) was added, giving the mixture a dark green color. The solution was stirred for 1.5 hrs at RT, after which time the color had dissipated to give a clear/colorless solution. Triphenylphosphine (11 mg, $0.041 \mathrm{mmol})$ was added to coordinate the borane catalyst. To the resulting solution, hexanes $(2 \mathrm{~mL})$ were added and the borane-phosphine adduct was observed to precipitate as a fine white suspension. The mixture was cooled at $-22^{\circ} \mathrm{C}$ for $0.5 \mathrm{hr}$, then filtered through a $0.5 \mathrm{~cm}$ plug of Celite in a Pasteur pipet. Removal of the volatiles from the mother liquor under reduced pressure afforded $\mathbf{1}$ as a white solid (445 mg, 97\%). Anal. Calc. for $\mathrm{C}_{31} \mathrm{H}_{26} \mathrm{SSi}$ : C 81.17, H 5.71; Found: C 81.29, H 5.69. ${ }^{1} \mathrm{H}$ NMR (250 MHz, $\left.\mathrm{C}_{6} \mathrm{D}_{6}\right) \delta 5.22\left(\mathrm{~s}, 1 \mathrm{H}, \mathrm{SC}_{\underline{\mathrm{HPh}}}\right), 6.95-7.19(\mathrm{~m}, 15 \mathrm{H}, \mathrm{Ar}), 7.29\left(\mathrm{~d}, \mathrm{~J}_{\mathrm{HH}}=7.0 \mathrm{~Hz}, 4 \mathrm{H}, \mathrm{Ar}\right), 7.68\left(\mathrm{dd}, \mathrm{J}_{\mathrm{HH}}=5.0\right.$ 
$\mathrm{Hz} / 2.5 \mathrm{~Hz}, 6 \mathrm{H}, \mathrm{Ar}) ;{ }^{13} \mathrm{C}\left\{{ }^{1} \mathrm{H}\right\} \mathrm{NMR}\left(62.90 \mathrm{MHz}, \mathrm{C}_{6} \mathrm{D}_{6}\right) \delta 51.48,126.87,128.23,128.49,128.54,130.28$, 133.56, 136.29, 144.46. ${ }^{29} \mathrm{Si}\left\{{ }^{1} \mathrm{H}\right\} \mathrm{NMR}\left(99.36 \mathrm{MHz}, \mathrm{C}_{6} \mathrm{D}_{6}\right)-1.84$. MS (ESI) $m / z: 458(\mathrm{M}), 199$ (M $\left.\mathrm{SiPh}_{3}\right), 167\left(\mathrm{M}-\mathrm{SSiPh}_{3}\right), 152,82,51$.

2:. Diphenylmethylsilane (151mg, $0.76 \mathrm{mmol})$ and thiobenzophenone (150 $\mathrm{mg}, 0.76 \mathrm{mmol})$ were combined in hexanes $(1.5 \mathrm{~mL})$. To the resulting strongly blue-colored solution, $\mathrm{B}\left(\mathrm{C}_{6} \mathrm{~F}_{5}\right)_{3}$ was added $(0.05$ $\mathrm{mg}, 9.6 \times 10^{-5} \mathrm{mmol}, 0.10 \mathrm{~mL}$ of $0.5 \mathrm{mg} / \mathrm{mL} \mathrm{B}\left(\mathrm{C}_{6} \mathrm{~F}_{5}\right)_{3} /$ hexanes solution) using a $1 \mathrm{~mL}$ graduated pipet. The mixture was stirred at RT for $3 \mathrm{hrs}$ until the color of the solution dissipated to a faint blue. The solvent was removed under reduced pressure to afford 2 as a light blue oil (298 mg, 99\%). Anal. Calc. for $\mathrm{C}_{26} \mathrm{H}_{24} \mathrm{SSi}$ : C 78.73, H 6.10; Found: C 78.93, H 5.75. ${ }^{1} \mathrm{H}$ NMR $\left(250 \mathrm{MHz}, \mathrm{C}_{6} \mathrm{D}_{6}\right) \delta 0.46$ (s, 3H, SiC $\left.\underline{H}_{3}\right)$, $5.12\left(\mathrm{~s}, 1 \mathrm{H}, \mathrm{SC}_{\mathrm{HPh}}\right), 6.91-7.11(\mathrm{~m}, 6 \mathrm{H}, \mathrm{Ar}), 7.11-7.21(\mathrm{~m}, 6 \mathrm{H}), 7.32\left(\mathrm{~d}, \mathrm{~J}_{\mathrm{HH}}=8.2 \mathrm{~Hz}, 4 \mathrm{H}, \mathrm{Ar}\right), 7.62(\mathrm{~m}$, $4 \mathrm{H}, \mathrm{Ar}) ;{ }^{13} \mathrm{C}\left\{{ }^{1} \mathrm{H}\right\} \operatorname{HMR}\left(62.90 \mathrm{MHz}, \mathrm{C}_{6} \mathrm{D}_{6}\right) \delta-1.30,51.60,127.41,128.68,128.92,128.97,130.59$, 135.52, 144.98. ${ }^{29} \mathrm{Si}\left\{{ }^{1} \mathrm{H}\right\}$ NMR (99.36 MHz, $\left.\mathrm{C}_{6} \mathrm{D}_{6}\right)$ 2.79. MS (ESI) $m / z: 396(\mathrm{M}), 167\left(\mathrm{M}-\mathrm{SSiMePh}_{2}\right)$, 152.

3: Triethylsilane (100 mg, $0.85 \mathrm{mmol})$ and thiobenzophenone $(169 \mathrm{mg}, 0.85 \mathrm{mmol})$ were combined in hexanes $(2 \mathrm{~mL})$. To the resulting strongly blue-colored solution, $\mathrm{B}\left(\mathrm{C}_{6} \mathrm{~F}_{5}\right)_{3}$ was added $\left(0.05 \mathrm{mg}, 1 \times 10^{-4}\right.$ mmol, $0.1 \mathrm{~mL}$ of $0.5 \mathrm{mg} / \mathrm{mL} \mathrm{B}\left(\mathrm{C}_{6} \mathrm{~F}_{5}\right)_{3} /$ hexanes solution) using a $1 \mathrm{~mL}$ graduated pipet. The mixture was stirred under static $\mathrm{N}_{2}$ for 4 hrs until the color changed to faint blue. The solvent was removed under reduced pressure to afford 4 as a lightly blue-tinged oil $(262 \mathrm{mg}, 98 \%)$. Anal. Calc. for $\mathrm{C}_{19} \mathrm{H}_{26} \mathrm{SSi}$ : C 72.52, H 8.35; Found: C 72.64, H 8.12. ${ }^{1} \mathrm{H}$ NMR $\left(250 \mathrm{MHz}, \mathrm{C}_{6} \mathrm{D}_{6}\right) \delta 0.60\left[\mathrm{q}, \mathrm{J}_{\mathrm{HH}}=7.7 \mathrm{~Hz}, 6 \mathrm{H}\right.$, $\left.\mathrm{Si}\left(\mathrm{C}_{\mathrm{H}_{2}} \mathrm{CH}_{3}\right)_{3}\right], 0.91\left[\mathrm{t}, \mathrm{J}_{\mathrm{HH}}=7.7 \mathrm{~Hz}, 9 \mathrm{H}, \mathrm{Si}\left(\mathrm{CH}_{2} \mathrm{CH}_{3}\right)_{3}\right], 5.21\left(\mathrm{~s}, 1 \mathrm{H}, \mathrm{SC} \underline{\mathrm{HPh}}{ }_{2}\right), 6.97\left[\mathrm{t}, \mathrm{J}_{\mathrm{HH}}=7.8 \mathrm{~Hz}, 2 \mathrm{H}\right.$, $\left.\mathrm{SCH}\left(\mathrm{p}-\mathrm{C}_{6} \underline{\mathrm{H}}_{5}\right)_{2}\right], 7.08\left[\mathrm{t}, \mathrm{J}_{\mathrm{HH}}=7.8 \mathrm{~Hz}, 6 \mathrm{H}, \mathrm{SCH}\left(\mathrm{m}-\mathrm{C}_{6} \underline{\mathrm{H}}_{5}\right)_{2}\right], 7.48\left[\mathrm{~d}, \mathrm{~J}_{\mathrm{HH}}=7.8 \mathrm{~Hz}, 4 \mathrm{H}, \mathrm{SCH}\left(\mathrm{o}-\mathrm{C}_{6} \underline{\mathrm{H}}_{5}\right)_{2}\right]$; ${ }^{13} \mathrm{C}\left\{{ }^{1} \mathrm{H}\right\} \operatorname{HMR}\left(62.90 \mathrm{MHz}, \mathrm{C}_{6} \mathrm{D}_{6}\right) \delta 5.93,7.79,50.12,127.39,128.89 .128 .95,145.51 .{ }^{29} \mathrm{Si}\left\{{ }^{1} \mathrm{H}\right\} \mathrm{NMR}$ (99.36 MHz, $\left.\mathrm{C}_{6} \mathrm{D}_{6}\right)$ 22.19. MS (ESI) $m / z: 314(\mathrm{M}), 167\left(\mathrm{M}-\mathrm{SSiEt}_{3}\right), 152$.

4: Dimethylphenylsilane (103 $\mathrm{mg}, 0.76 \mathrm{mmol})$ and thiobenzophenone (150 $\mathrm{mg}, 0.76 \mathrm{mmol})$ were combined in hexanes $(1.5 \mathrm{~mL})$. To the resulting strongly blue-colored solution, $\mathrm{B}\left(\mathrm{C}_{6} \mathrm{~F}_{5}\right)_{3}$ was added $(0.03$

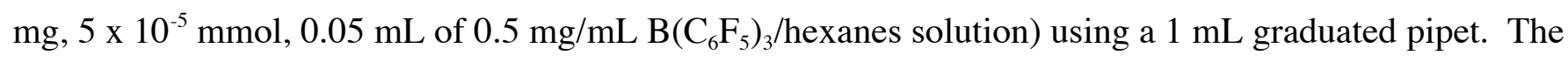
mixture was stirred under static $\mathrm{N}_{2}$ for $0.5 \mathrm{hr}$ until the color changed to faint blue. The solvent was removed under reduced pressure to afford 3 as a light blue oil $(251 \mathrm{mg}, 99 \%)$. Anal. Calc. for $\mathrm{C}_{21} \mathrm{H}_{22} \mathrm{SSi}$ :

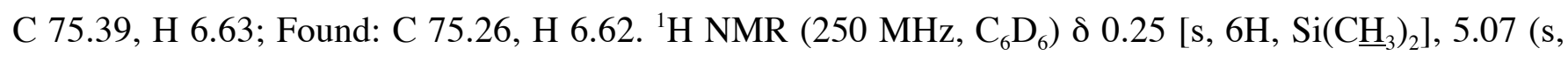

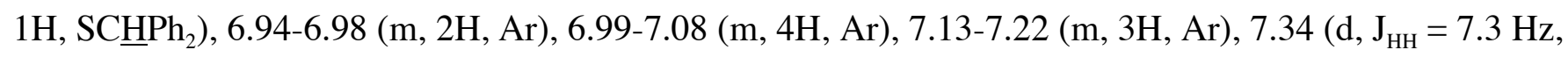
$4 \mathrm{H}, \mathrm{Ar}), 7.51-7.58$ (m, 2H, Ar); ${ }^{13} \mathrm{C}\left\{{ }^{1} \mathrm{H}\right\} \mathrm{HMR}\left(62.90 \mathrm{MHz}, \mathrm{C}_{6} \mathrm{D}_{6}\right) \delta-0.07,51.33,127.37,128.60$, 128.91, 128.95, 130.42, 134.74, 137.11, 144.96. ${ }^{29} \mathrm{Si}\left\{{ }^{1} \mathrm{H}\right\}$ NMR $\left(99.36 \mathrm{MHz}, \mathrm{C}_{6} \mathrm{D}_{6}\right)$ 8.47. MS (ESI) $m / z$ : $167\left(\mathrm{M}-\mathrm{SSiMe}_{2} \mathrm{Ph}\right), 152$.

5: 1,1,2,2-tetramethyldisilane (90 $\mathrm{mg}, 0.76 \mathrm{mmol})$ and thiobenzophenone (300 $\mathrm{mg}, 1.51 \mathrm{mmol})$ were combined in toluene $(4 \mathrm{~mL})$. To the resulting strongly blue-colored solution, $\mathrm{B}\left(\mathrm{C}_{6} \mathrm{~F}_{5}\right)_{3}$ was added $(0.03$ 
$\mathrm{mg}, 6.6 \times 10^{-5} \mathrm{mmol}, 0.07 \mathrm{~mL}$ of $0.5 \mathrm{mg} / \mathrm{mL} \mathrm{B}\left(\mathrm{C}_{6} \mathrm{~F}_{5}\right)_{3} /$ toluene solution) using a $1 \mathrm{~mL}$ graduated pipet. The mixture was stirred under static $\mathrm{N}_{2}$ for approx. $0.5 \mathrm{hr}$ until the blue color dissipated to clear/colorless. The solvent was removed under reduced pressure to afford a white solid. This material was dissolved in toluene $(0.5 \mathrm{~mL})$ and precipitated, at $-22^{\circ} \mathrm{C}$, with hexanes $(1.5 \mathrm{~mL})$ to yield 5 as white needles $(356 \mathrm{mg}$, 91\%). Anal. Calc. for $\mathrm{C}_{30} \mathrm{H}_{34} \mathrm{~S}_{2} \mathrm{Si}_{2}$ : C 69.98, H 6.66; Found: C 70.00, H 7.04. ${ }^{1} \mathrm{H}$ NMR (300 MHz, $\left.\mathrm{C}_{6} \mathrm{D}_{6}\right) \delta$ $0.22\left\{\mathrm{~s}, 12 \mathrm{H},\left[\mathrm{Si}\left(\mathrm{CH}_{3}\right)_{2}\right]_{2}\right\}, 5.41\left[\mathrm{~s}, 2 \mathrm{H}, \mathrm{SC}_{\underline{H P h}}(\mathrm{x} 2)\right], 6.99\left[\mathrm{t}, \mathrm{J}_{\mathrm{HH}}=7.6 \mathrm{~Hz}, 4 \mathrm{H}, \mathrm{SCH}\left(\mathrm{p}-\mathrm{C}_{6} \underline{\mathrm{H}}_{5}\right)_{2}\right], 7.06[\mathrm{t}$, $\left.\mathrm{J}_{\mathrm{HH}}=7.2 \mathrm{~Hz}, 8 \mathrm{H}, \mathrm{SCH}\left(\mathrm{m}_{-} \mathrm{C}_{6} \underline{\mathrm{H}}_{5}\right)_{2}\right], 7.50\left[\mathrm{~d}, \mathrm{~J}_{\mathrm{HH}}=7.2 \mathrm{~Hz}, 8 \mathrm{H}, \mathrm{SCH}\left(\mathrm{o}-\mathrm{C}_{6} \underline{\mathrm{H}}_{5}\right)_{2}\right] ;{ }^{13} \mathrm{C}\left\{{ }^{1} \mathrm{H}\right\} \mathrm{HMR}(75.47 \mathrm{MHz}$, $\left.\mathrm{C}_{6} \mathrm{D}_{6}\right) \delta-0.58,51.27,127.50,128.99,129.03,145.15 .{ }^{29} \mathrm{Si}\left\{{ }^{1} \mathrm{H}\right\} \mathrm{NMR}\left(99.36 \mathrm{MHz}, \mathrm{C}_{6} \mathrm{D}_{6}\right)-2.23$. MS (ESI) $m / z: 199\left[\mathrm{M}-\mathrm{Me}_{2} \mathrm{SiSiMe}_{2}\left(\mathrm{SCHPh}_{2}\right)\right], 167$ [M - Me $\left.\mathrm{Si}_{2}(\mathrm{~S}) \mathrm{SiMe}_{2}\left(\mathrm{SCHPh}_{2}\right)\right], 121,105,69,55$.

6: 1,1,2,2-tetraphenyldisilane (179 $\mathrm{mg}, 0.48 \mathrm{mmol})$ and thiobenzophenone (97 $\mathrm{mg}, 0.49 \mathrm{mmol})$ were combined with hexanes $(4 \mathrm{~mL}) . \mathrm{B}\left(\mathrm{C}_{6} \mathrm{~F}_{5}\right)_{3}(10 \mathrm{mg}, 0.020 \mathrm{mmol}, 4 \mathrm{~mol} \%)$ was added to this blue solution. The mixture was stirred under static $\mathrm{N}_{2}$ for $3 \mathrm{hrs}$ until the solution was clear and colorless. Triphenylphosphine $(5 \mathrm{mg}, 0.21 \mathrm{mmol})$ was added to coordinate the borane catalyst. The boranephosphine adduct was observed to precipitate as a fine white suspension in hexanes. The mixture was cooled at $-22^{\circ} \mathrm{C}$ for $0.5 \mathrm{hr}$, then filtered through a $1.5 \mathrm{~cm}$ diameter glass frit funnel with the aid of vacuum. The volatiles were removed under high vacuum (whilst heating at $80^{\circ} \mathrm{C}$ for $12 \mathrm{hrs}$ ) from the mother liquor to give 6 as a clear/colorless viscous oil ( $257 \mathrm{mg}, 95 \%$ ). Anal. Calc. for $\mathrm{C}_{37} \mathrm{H}_{32} \mathrm{SSi}_{2}: \mathrm{C}$ 78.66, H 5.72; Found: C 78.40, H 5.52. ${ }^{1} \mathrm{H}$ NMR (250 MHz, $\left.\mathrm{C}_{6} \mathrm{D}_{6}\right) \delta 5.25\left(\mathrm{~s}, 1 \mathrm{H}, \mathrm{SCHPh}_{2}\right), 5.49(\mathrm{~s}, 1 \mathrm{H}$, $\mathrm{SiH}, \mathrm{Ar}), 6.87-7.12(\mathrm{~m}, 18 \mathrm{H}, \mathrm{Ar}), 7.25(\mathrm{dd}, 7.8 \mathrm{~Hz} / 1.8 \mathrm{~Hz}, 4 \mathrm{H}, \mathrm{Ar}), 7.57-7.67$ (m, 8H, Ar); ${ }^{13} \mathrm{C}\left\{{ }^{1} \mathrm{H}\right\}$ HMR $\left(62.90 \mathrm{MHz}, \mathrm{C}_{6} \mathrm{D}_{6}\right) \delta 52.20,127.27,128.68,128.87,128.98,130.20,130.48,132.85,136.71$, 137.03, 144.77. ${ }^{29} \mathrm{Si}\left\{{ }^{1} \mathrm{H}\right\}$ NMR (99.36 MHz, $\mathrm{C}_{6} \mathrm{D}_{6}$ ) -9.93, -36.26 (inverted). MS (ESI) m/z: 294, 182 [M $\left.-\mathrm{Ph}_{2} \mathrm{SiH}\left(\mathrm{SCHPh}_{2}\right)\right], 167\left[\mathrm{M}-\mathrm{Ph}_{2} \mathrm{Si}(\mathrm{S}) \mathrm{SiPh}_{2}\left(\mathrm{SCHPh}_{2}\right)\right], 152,83,69$.

Ethanolysis reactions of 1-6 Each silylthiother substrate $(0.75 \mathrm{mmol})$ was mixed with ethanol (dried/distilled over $\left.\mathrm{Mg} / \mathrm{I}_{2}\right)\left(1.5 \mathrm{~mL}\right.$ ) and stirred under $\mathrm{N}_{2}(\mathrm{~g})$ open to a nujol bubbler. See Table 1 for individual reaction times/conditions. The reactions were monitored by ${ }^{1} \mathrm{H}$ NMR spectroscopy (see assignments below).

Table 1. Ethanolysis reactions ${ }^{\hat{a}}$

\begin{tabular}{|c|c|c|}
\hline $\mathrm{R}_{2} \mathrm{RSi}_{-} \mathrm{SCHPh}_{2}$ & $\stackrel{\text { excess EtOH }}{\longrightarrow}$ & \multirow[t]{2}{*}{$+\quad \mathrm{H}-\mathrm{SCHPh}$} \\
\hline Substrate & Reaction time/conditions & \\
\hline 1 & $20 \mathrm{hrs} / \mathrm{reflux}$ in EtOH & $\mathrm{Ph}_{3} \mathrm{SiOEt}+\mathrm{HSCHPh}_{2}$ \\
\hline 2 & $20 \mathrm{hrs} / \mathrm{RT}$ in EtOH & $\mathrm{Ph}_{2} \mathrm{MeSiOEt}+\mathrm{HSCHPh}_{2}$ \\
\hline $3^{b}$ & $20 \mathrm{hrs} / \mathrm{RT}$ in $\mathrm{EtOH}$ & $\mathrm{Et}_{3} \mathrm{SiOEt}+\mathrm{HSCHPh}_{2}$ (70\% conv.) \\
\hline 4 & $16 \mathrm{hrs} / \mathrm{RT}$ in EtOH & $\mathrm{PhMe}_{2} \mathrm{SiOEt}+\mathrm{HSCHPh}{ }_{2}$ \\
\hline $5^{b}$ & $20 \mathrm{hrs} / \mathrm{reflux}$ in $\mathrm{EtOH}$ & $\left(\mathrm{Me}_{2} \mathrm{SiOEt}\right)_{2}+\mathrm{HSCHPh}_{2}$ \\
\hline 6 & $25 \mathrm{hrs} / \mathrm{reflux}$ in $\mathrm{EtOH}$ & $\begin{array}{l}\mathrm{Ph}_{2}(\mathrm{H}) \mathrm{Si}_{-} \mathrm{SiPh}_{2}(\mathrm{OEt})(50 \% \text { conv. })+ \\
\mathrm{HSCHPh}_{2}\end{array}$ \\
\hline
\end{tabular}


$1\left(\mathrm{Ph}_{3} \mathrm{SiSCHPh}_{2}\right)+\mathrm{EtOH}:{ }^{1} \mathrm{H} \mathrm{NMR}\left(250 \mathrm{MHz}, \mathrm{C}_{6} \mathrm{D}_{6}\right) \delta 1.13\left(\mathrm{t}, \mathrm{J}_{\mathrm{HH}}=7.7 \mathrm{~Hz}, 3 \mathrm{H}, \mathrm{SiOCH}_{2} \underline{\mathrm{H}}_{3}\right), 1.97(\mathrm{~d}$, $\left.\mathrm{J}_{\mathrm{HH}}=7.5 \mathrm{~Hz}, 1 \mathrm{H}, \underline{\mathrm{HS}}\right), 3.68\left(\mathrm{q}, \mathrm{J}_{\mathrm{HH}}=7.7 \mathrm{~Hz}, 2 \mathrm{H}, \mathrm{SiOC}_{2} \mathrm{CH}_{3}\right), 5.16\left(\mathrm{~d}, \mathrm{~J}_{\mathrm{HH}}=7.5 \mathrm{~Hz}, 1 \mathrm{H}, \mathrm{HSC} \underline{\mathrm{H}}\right), 6.78-$ 7.42 (m, 12H, Ar), 7.59-7.91 (m, 3H, Ar).

$2\left(\mathrm{Ph}_{2} \mathrm{MeSiSCHPh}_{2}\right)+\mathrm{EtOH}:{ }^{1} \mathrm{H}$ NMR $\left(250 \mathrm{MHz}, \mathrm{C}_{6} \mathrm{D}_{6}\right) \delta 0.12\left(\mathrm{~s}, 3 \mathrm{H}, \mathrm{SiC}_{3}\right), 1.12\left(\mathrm{t}, \mathrm{J}_{\mathrm{HH}}=7.7 \mathrm{~Hz}, 3 \mathrm{H}\right.$, $\left.\mathrm{SiOCH}_{2} \underline{\mathrm{CH}}_{3}\right), 1.97\left(\mathrm{~d}, \mathrm{~J}_{\mathrm{HH}}=7.5 \mathrm{~Hz}, 1 \mathrm{H}, \underline{\mathrm{H} S}\right), 3.67\left(\mathrm{q}, \mathrm{J}_{\mathrm{HH}}=7.7 \mathrm{~Hz}, 2 \mathrm{H}, \mathrm{SiOC}_{2} \mathrm{CH}_{3}\right), 5.16\left(\mathrm{~d}, \mathrm{~J}_{\mathrm{HH}}=7.5\right.$ Hz, 1H, HSC $\underline{H}$ ), 6.92-7.09 (m, 6H, Ar), 7.12-7.23 (m, 6H, Ar), 7.24-7.30 (m, 4H, Ar), 7.61-7.69 (m, 4H, $\operatorname{Ar})$.

$3\left(\mathrm{PhMe}_{2} \mathrm{SiSCHPh}_{2}\right)+\mathrm{EtOH}$ : **Note that alkoxysilane product $\mathrm{PhMe}_{2} \mathrm{SiOEt}$ is mildly volatile so integration ratios are not exact. ${ }^{1} \mathrm{H} \mathrm{NMR}\left(250 \mathrm{MHz}, \mathrm{C}_{6} \mathrm{D}_{6}\right) \delta 0.31\left[\mathrm{~s}, 6 \mathrm{H}, \mathrm{Si}\left(\mathrm{C}_{3}\right)_{2}\right], 1.10\left(\mathrm{t}, \mathrm{J}_{\mathrm{HH}}=7.7 \mathrm{~Hz}\right.$, $3 \mathrm{H}, \mathrm{SiOCH}_{2} \underline{\mathrm{CH}}_{3}$ ), $1.97\left(\mathrm{~d}, \mathrm{~J}_{\mathrm{HH}}=7.5 \mathrm{~Hz}, 1 \mathrm{H}, \underline{\mathrm{HS}}\right), 3.55\left(\mathrm{q}, \mathrm{J}_{\mathrm{HH}}=7.7 \mathrm{~Hz}, 2 \mathrm{H}, \mathrm{SiOC}_{2} \mathrm{CH}_{3}\right), 5.11\left(\mathrm{~d}, \mathrm{~J}_{\mathrm{HH}}=\right.$

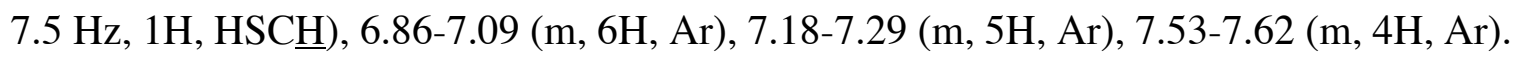

$4\left(\mathrm{Et}_{3} \mathrm{SiSCHPh}_{2}\right)+\mathrm{EtOH}: * *$ Note that the alkoxysilane product $\mathrm{Et}_{3} \mathrm{SiOEt}$ is volatile and is not observed directly by NMR. The following NMR data shows thiol (solvolysis byproduct) and unreacted thiosilane. Consequently, integration ratios are not reported. ${ }^{1} \mathrm{H} \mathrm{NMR}\left(250 \mathrm{MHz}, \mathrm{C}_{6} \mathrm{D}_{6}\right) \delta 0.60\left[\mathrm{q}, \mathrm{J}_{\mathrm{HH}}=7.7 \mathrm{~Hz}\right.$, $\left.\mathrm{Si}\left(\mathrm{CH}_{2} \mathrm{CH}_{3}\right)_{3}\right], 0.91\left[\mathrm{t}, \mathrm{J}_{\mathrm{HH}}=7.7 \mathrm{~Hz}, \mathrm{Si}\left(\mathrm{CH}_{2} \underline{\mathrm{CH}}_{3}\right)_{3}\right], 1.97\left(\mathrm{~d}, \mathrm{~J}_{\mathrm{HH}}=7.5 \mathrm{~Hz}, \underline{\mathrm{HS}}\right), 5.11\left(\mathrm{~d}, \mathrm{~J}_{\mathrm{HH}}=7.5 \mathrm{~Hz}\right.$, HSC $\underline{H}$ ), 5.22 [s, 1H, SiSC $\underline{H}$ (unreacted 4)], 6.84-7.15 (m, Ar), 7.24 (d, J $\left.\mathrm{J}_{\mathrm{HH}}=7.8 \mathrm{~Hz}, \mathrm{Ar}\right), 7.43\left(\mathrm{~d}, \mathrm{~J}_{\mathrm{HH}}=\right.$ $7.8 \mathrm{~Hz}, \mathrm{Ar})$. An approximate 1:2 integration ratio for $\mathrm{SiSC} \underline{\mathrm{H}}: \mathrm{S} \underline{\mathrm{H}}$ indicates $70 \%$ conversion for the conditions employed (Table 1).

$5\left[\left(\mathrm{Me}_{2} \mathrm{SiSCHPh}_{2}\right)_{2}\right]+\mathrm{EtOH}: * *$ Note that the alkoxysilane product $\left(\mathrm{Me}_{3} \mathrm{SiOEt}\right)_{2}$ is volatile and is not observed directly by NMR. Analysis of the reaction mixture showed only thiol. ${ }^{1} \mathrm{H}$ NMR $(250 \mathrm{MHz}$, $\left.\mathrm{C}_{6} \mathrm{D}_{6}\right) \delta 1.97\left(\mathrm{~d}, \mathrm{~J}_{\mathrm{HH}}=7.5 \mathrm{~Hz}, \underline{\mathrm{H} S}\right), 5.11\left(\mathrm{~d}, \mathrm{~J}_{\mathrm{HH}}=7.5 \mathrm{~Hz}, \mathrm{HSC} \underline{\mathrm{H}}\right), 6.92-7.15\left[\mathrm{~m}, 6 \mathrm{H}, \mathrm{SCH}\left(\mathrm{m}, \mathrm{p}-\mathrm{C}_{6} \underline{\mathrm{H}}_{5}\right)_{2}\right]$, $7.27\left[\mathrm{~d}, \mathrm{~J}_{\mathrm{HH}}=7.8 \mathrm{~Hz}, 4 \mathrm{H}, \mathrm{SCH}\left(\mathrm{o}-\mathrm{C}_{6} \underline{\mathrm{H}}_{5}\right)_{2}\right]$.

$6\left[\mathrm{Ph}_{2} \mathrm{Si}(\mathrm{H}) \mathrm{SiPh}_{2}\left(\mathrm{SCHPh}_{2}\right)\right]+\mathrm{EtOH}:{ }^{1} \mathrm{H} \mathrm{NMR}\left(250 \mathrm{MHz}, \mathrm{C}_{6} \mathrm{D}_{6}\right) \delta 1.09\left(\mathrm{t}, \mathrm{J}_{\mathrm{HH}}=7.7 \mathrm{~Hz}, \mathrm{SiOCH}_{2} \mathrm{C}_{3}\right)$, $1.97\left(\mathrm{~d}, \mathrm{~J}_{\mathrm{HH}}=7.5 \mathrm{~Hz}, \underline{\mathrm{HS}}\right), 3.75\left(\mathrm{q}, \mathrm{J}_{\mathrm{HH}}=7.7 \mathrm{~Hz}, \mathrm{SiOCH}_{2} \mathrm{CH}_{3}\right), 5.11\left(\mathrm{~d}, \mathrm{~J}_{\mathrm{HH}}=7.5 \mathrm{~Hz}, \mathrm{HSC} \underline{\mathrm{H}}\right), 5.24(\mathrm{~s}$, $\mathrm{SC} \underline{\mathrm{H}}$ ), 5.48 [s, Siㅆ (starting material)], 5.55 [s, SiH (product)], 6.80-7.27 (m, Ar), 7.56-7.62 (m, Ar). An approximate 1:1 integration ratio for $\mathrm{Si} \underline{\mathrm{H}}$ (starting material):S $\underline{\mathrm{H}}$ (product) indicates $50 \%$ conversion for the conditions employed (Table 1).

FT-IR analysis of an admixture of $B\left(\mathbf{C}_{6} \mathbf{F}_{5}\right)_{3}, \mathbf{O}=\mathrm{CPh}_{2}$ and $\mathrm{S}=\mathrm{CPh}_{2}$ : By analytical pipet, $0.5 \mathrm{~mL}$ of standardized solutions $\left(0.10 \mathrm{M}\right.$ in benzene) of $\mathrm{B}(\mathrm{C} 6 \mathrm{~F} 5)_{3}, \mathrm{O}=\mathrm{CPh}_{2}$ and $\mathrm{S}=\mathrm{CPh}_{2}$ were combined and diluted to $2 \mathrm{~mL}$, thus giving a fixed, equimolar concentration of $0.025 \mathrm{M}$ for each analyte. From the resulting dark blue solution, an aliquot was removed and placed in a quartz solution cell and sealed for FT-IR analysis.

When $\mathrm{O}=\mathrm{CPh}_{2}$ is combined with $\mathrm{B}\left(\mathrm{C}_{6} \mathrm{~F}_{5}\right)_{3}$ in a $1: 1$ ratio, the carbonyl stretch at $1660 \mathrm{~cm}^{-1}$ is not observed, implying that the carbonyl molecules are engaged by the borane. However, upon addition of an 
equivalent of $\mathrm{S}=\mathrm{CPh}_{2}$ (to give a $1: 1: 1 \mathrm{~B}\left(\mathrm{C}_{6} \mathrm{~F}_{5}\right)_{3} / \mathrm{O}=\mathrm{CPh}_{2} / \mathrm{S}=\mathrm{CPh}_{2}$ mixture) a weak carbonyl peak becomes apparent (Fig. 1).

The implication is that the small amount of free carbonyl observed corresponds to an equimolar amount of $\mathrm{S}=\mathrm{CPh}_{2}$ complexed by borane, while the bulk of the borane remains complexed with the ketone. This suggests that the majority of the thioketone molecules exist in an uncomplexed state, corresponding to the number of $\mathrm{Ph}_{2} \mathrm{C}=\mathrm{O}$ molecules engaged by the borane. A control experiment was also conducted to assess the effect of $\mathrm{S}=\mathrm{CPh}_{2}$ on the $\mathrm{CO}$ stretch of $\mathrm{O}=\mathrm{CPh}_{2}$ (Table 1, iv-a). As expected, the impact was found to be negligible.

Table 1. Selected FT-IR data for competitive binding experiments

\begin{tabular}{|l|l|l|l|}
\hline & Analyte $(\mathrm{s})$ & Signal of interest & Appearance of signal \\
\cline { 1 - 1 } i-a & $\mathrm{O}=\mathrm{CPh}_{2}$ & $\mathrm{O}=\mathrm{C} \operatorname{str}\left(1660 \mathrm{~cm}^{-1}\right)$ & Strong \\
\cline { 1 - 1 } iii-a & $\mathrm{O}=\mathrm{CPh}_{2}+\mathrm{B}\left(\mathrm{C}_{6} \mathrm{~F}_{5}\right)_{3}$ & $\mathrm{O}=\mathrm{C} \operatorname{str}\left(1660 \mathrm{~cm}^{-1}\right)$ & Not observed \\
\cline { 1 - 1 } iii-a & $\mathrm{O}=\mathrm{CPh}_{2}+\mathrm{B}\left(\mathrm{C}_{6} \mathrm{~F}_{5}\right)_{3}+\mathrm{S}=\mathrm{CPh}_{2}$ & $\mathrm{O}=\mathrm{C} \operatorname{str}\left(1660 \mathrm{~cm}^{-1}\right)$ & Weak \\
\cline { 1 - 1 } iv-a & $\mathrm{O}=\mathrm{CPh}_{2}+\mathrm{S}=\mathrm{CPh}_{2}$ & $\mathrm{O}=\mathrm{C} \operatorname{str}\left(1660 \mathrm{~cm}^{-1}\right)$ & Strong [same as (i-a)] \\
\cline { 1 - 2 } & & &
\end{tabular}

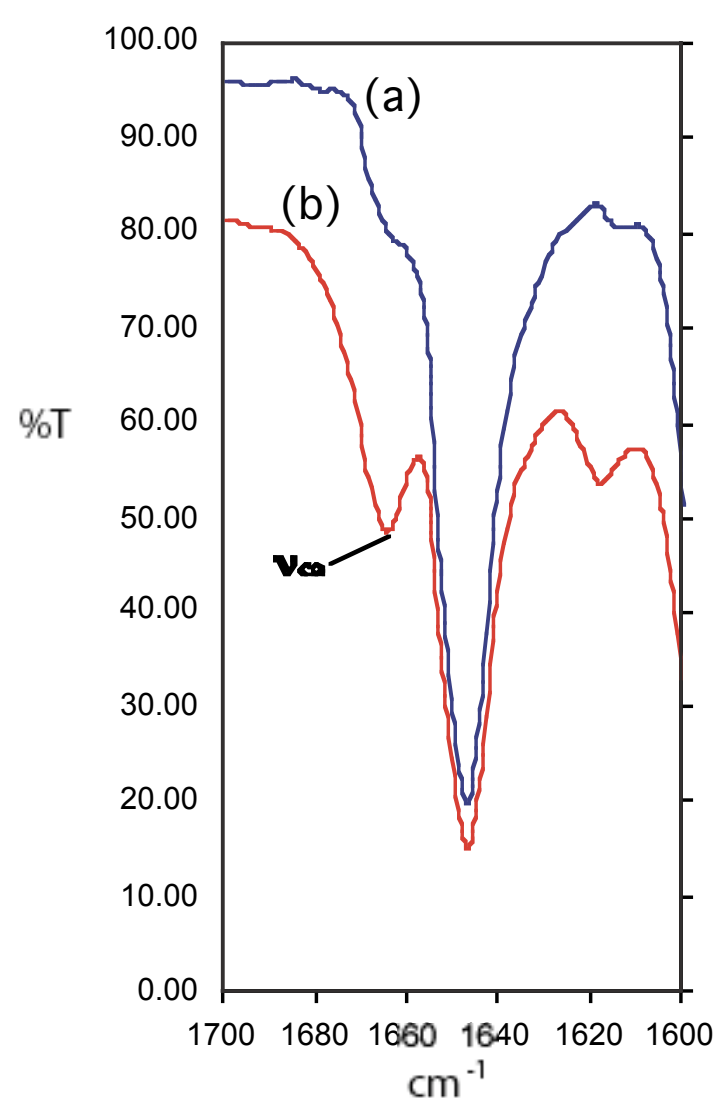

Fig.1 Infrared results from ketone/thioketone competitive borane-bonding experiments. (a) $\mathrm{B}\left(\mathrm{C}_{6} \mathrm{~F}_{5}\right)_{3} / \mathrm{O}=\mathrm{CPh}_{2}$ (b) $\mathrm{B}\left(\mathrm{C}_{6} \mathrm{~F}_{5}\right)_{3} / \mathrm{O}=\mathrm{CPh}_{2} / \mathrm{S}=\mathrm{CPh}_{2}$, all $0.025 \mathrm{M}$ in benzene. (Traces offset by $\sim 17 \% \mathrm{~T}$ for clarity.) 
UV-vis analysis of an admixture of $\mathbf{B}\left(\mathrm{C}_{6} \mathbf{F}_{5}\right)_{3}, \mathbf{O}=\mathrm{CPh}_{2}$ and $\mathrm{S}=\mathrm{CPh}_{2}$ : By analytical pipet, $0.5 \mathrm{~mL}$ of standardized solutions (0.019 $\mathrm{M}$ in benzene) of $\mathrm{B}(\mathrm{C} 6 \mathrm{~F} 5)_{3}, \mathrm{O}=\mathrm{CPh}_{2}$ and $\mathrm{S}=\mathrm{CPh}_{2}$ were combined and diluted to $2.5 \mathrm{~mL}$, thus giving a fixed, equimolar concentration of $0.0038 \mathrm{M}$ for each analyte. The resulting solution was placed in a quartz UV-vis cell and sealed for UV-vis analysis.

As seen in Table 2, free $\mathrm{Ph}_{2} \mathrm{C}=\mathrm{S}$ has a local maximum absorption at approximately $603 \mathrm{~nm}$, with a molar absorptivity of $230 \mathrm{M}^{-1} \mathrm{~cm}^{-1}$. When $\mathrm{B}\left(\mathrm{C}_{6} \mathrm{~F}_{5}\right)_{3}$ is added (i.e. $1: 1 \mathrm{~B}\left(\mathrm{C}_{6} \mathrm{~F}_{5}\right)_{3} / \mathrm{Ph}_{2} \mathrm{C}=\mathrm{S}$ ), the intensity of this signal is diminished $\left(\varepsilon=160 \mathrm{M}^{-1} \mathrm{~cm}^{-1}\right)$. In the case or the $1: 1: 1 \mathrm{~B}\left(\mathrm{C}_{6} \mathrm{~F}_{5}\right)_{3} / \mathrm{Ph}_{2} \mathrm{C}=\mathrm{S} / \mathrm{Ph}_{2} \mathrm{C}=\mathrm{O}$ system, the intensity of absorption is close to that of free $\mathrm{Ph}_{2} \mathrm{C}=\mathrm{S}\left(\varepsilon=210 \mathrm{M}^{-1} \mathrm{~cm}^{-1}\right)$. From these data, we infer that, in the presence of thiocarbonyl and carbonyl moieties, the borane is preferentially complexed by the carbonyl. However, a small (but detectable - also seen by IR analysis) amount of $\mathrm{Ph}_{2} \mathrm{C}=\mathrm{S}$ competes in this equilibrium, causing a minor perturbation of the $\mathrm{S}=\mathrm{C} n \rightarrow \pi^{*}$ signal. Again, a $1: 1 \mathrm{Ph}_{2} \mathrm{C}=\mathrm{S} / \mathrm{Ph}_{2} \mathrm{C}=\mathrm{O}$ experiment was carried out as a control to ensure that benzophenone does not appreciably affect the $\mathrm{S}=\mathrm{C}$ UV-vis absorption. As expected, this mixture gives a UV-vis signal commensurate with the free thiocarbonyl case $\left(\varepsilon=220 \mathrm{M}^{-1} \mathrm{~cm}^{-1}\right)$.

Table 2. Selected UV-vis data for competitive binding experiments

\begin{tabular}{|c|c|c|c|c|c|}
\hline & Analyte(s) & $\begin{array}{l}\text { Transition of } \\
\text { interest }\end{array}$ & $\begin{array}{l}\lambda_{\max } \\
(\mathrm{nm})\end{array}$ & Abs & $\begin{array}{l}\varepsilon \\
\left(\mathrm{M}^{-1} \mathrm{~cm}^{-1}\right)\end{array}$ \\
\hline i-b & $\mathrm{S}=\mathrm{CPh}_{2}$ & $\mathrm{~S}=\mathrm{C} \mathrm{n} \rightarrow \pi^{*}$ & 603 & 0.86 & 230 \\
\hline ii-b & $\mathrm{S}=\mathrm{CPh}_{2}+\mathrm{B}\left(\mathrm{C}_{6} \mathrm{~F}_{5}\right)_{3}$ & $\mathrm{~S}=\mathrm{C} \mathrm{n} \rightarrow \pi^{*}$ & 604 & 0.60 & 160 \\
\hline iii-b & $\mathrm{S}=\mathrm{CPh}_{2}+\mathrm{B}\left(\mathrm{C}_{6} \mathrm{~F}_{5}\right)_{3}+\mathrm{O}=\mathrm{CPh}_{2}$ & $\mathrm{~S}=\mathrm{C} \mathrm{n} \rightarrow \pi^{*}$ & 602 & 0.79 & 210 \\
\hline iv-b & $\mathrm{O}=\mathrm{CPh}_{2}+\mathrm{S}=\mathrm{CPh}_{2}$ & $\mathrm{~S}=\mathrm{C} n \rightarrow \pi^{*}$ & 603 & 0.82 & 220 \\
\hline
\end{tabular}

Preliminary results for catalytic hydrosilylation of benzophenone

Hydrosilylation of benzophenone by $\mathrm{Ph}_{3} \mathrm{SiH}_{3}\left(0.5 \mathrm{~mol} \% \mathrm{~B}\left(\mathrm{C}_{6} \mathrm{~F}_{5}\right)_{3}\right)$ : Triphenylsilane (34 mg, $\left.0.19 \mathrm{mmol}\right)$ and benzophenone $(38 \mathrm{mg}, 0.19 \mathrm{mmol})$ were combined in benzene- $\mathrm{d}_{6}(1.5 \mathrm{~mL})$. To this clear, colourless solution, $\mathrm{B}\left(\mathrm{C}_{6} \mathrm{~F}_{5}\right)_{3}\left(0.5 \mathrm{mg}, 9.8 \times 10^{-4} \mathrm{mmol}, 0.10 \mathrm{~mL}\right.$ of $5 \mathrm{mg} / \mathrm{mL}$ solution of borane in $\left.\mathrm{C}_{6} \mathrm{D}_{6}\right)$ was added. The reaction was complete in $1.5 \mathrm{hrs}$ as determined by ${ }^{1} \mathrm{H}$ NMR. ${ }^{1} \mathrm{H}$ NMR $\left(250 \mathrm{MHz}, \mathrm{C}_{6} \mathrm{D}_{6}\right) \delta 6.09$ (s, $\left.1 \mathrm{H}, \mathrm{SiOC} \underline{H P h}_{2}\right), 6.95-7.20(\mathrm{~m}, 15 \mathrm{H}, \mathrm{Ar}), 7.37\left[\mathrm{~d}, \mathrm{~J}_{\mathrm{HH}}=7.8 \mathrm{~Hz}, 4 \mathrm{H}, \mathrm{OCH}\left(\mathrm{o}-\mathrm{C}_{6} \underline{\mathrm{H}}_{5}\right)_{2}\right], 7.69\left[\mathrm{~d}, \mathrm{~J}_{\mathrm{HH}}=7.8\right.$ $\left.\mathrm{Hz}, 6 \mathrm{H}, \mathrm{Si}\left(\mathrm{o}-\mathrm{C}_{6} \underline{\mathrm{H}}_{5}\right)_{3}\right]$

Selective hydrosilylation of benzophenone in the presence of thiobenzophenone using triphenylsilane ( 2 mol\% $\left.\mathrm{B}\left(\mathrm{C}_{6} \mathrm{~F}_{5}\right)_{3}\right)$ : Triphenylsilane (38 $\mathrm{mg}, 0.19 \mathrm{mmol}$ ), benzophenone (34 $\mathrm{mg}, 0.19 \mathrm{mmol}$ ) and thiobenzophenone $(38 \mathrm{mg}, 0.19 \mathrm{mmol})$ were combined in benzene- $\mathrm{d}_{6}(1.5 \mathrm{~mL})$. To this blue solution, $\mathrm{B}\left(\mathrm{C}_{6} \mathrm{~F}_{5}\right)_{3}\left(2 \mathrm{mg}, 3.9 \times 10^{-3} \mathrm{mmol}\right)$ was added. The reaction was complete in $<1 \mathrm{hr}\left(\right.$ by $\left.{ }^{1} \mathrm{H} \mathrm{NMR}\right)$. 


\section{References}

(1) Rosenberg, L.; Davis, C. W.; Yao, J. J. Am. Chem. Soc. 2001, 123, 5120.

(2) Massey, A. L.; Park, A. J. J. Organomet. Chem. 1964, 2, 245.

(3) (a) Cavaand, M. P.; Levinson, M. I. Tetrahedron 1985, 41, 5061. For an improved synthesis, see (b) Varma, R. S.; Kumar, D. Org. Lett. 1999, 1, 697. 